\title{
Planning Tracking Motion Insusceptible to Unknown Disturbances Using Sensitivity Measure
}

\author{
Tasuku Yamawaki and Masahito Yashima
}

\begin{abstract}
In the present paper, we show a novel motion planning method, which finds an arm trajectory that is insusceptible to unknown disturbances in the direction where task accuracy is required. First, we show that the sensitivity of the endeffector to unknown disturbances is measured by the direction of singular vectors and the condition number of a matrix which represents the input-output relation of the robotic arm system. We propose a motion planning method for tracking tasks, which finds the arm posture not only so that the maximum singular vector points toward the direction of a desired end-effector's path but so that the condition number becomes larger. We show by simulations and experiments that the end-effector can track the desired path as closely as possible even though disturbances are applied to the robotic arm.
\end{abstract}

\section{INTRODUCTION}

In tracking tasks such as welding and painting shown in Fig. 1, the end-effector of a robotic arm is required to follow a specified path on a workpiece while keeping a specified relative distance between a tool installed in the end-effector and the workpiece. In a practical robotic arm, friction, mechanical backlash, etc. are applied to the robotic arm as disturbances, which give rise to a collision with the workpiece and to a deterioration of the finishing state.

The trajectory tracking control problem of the robotic arm to suppress disturbances has been studied using different techniques, such as $H_{\infty}$ optimal control [2] and robust control [9]. In general, the design of these feedback controller systems is very complicated.

In order to develop a robust tracking system for the robotic arm, we consider incorporating motion planning techniques, which find an arm trajectory that is insusceptible to unknown disturbances, into conventional closed-loop systems. It enables us to obtain robust tracking systems with a simple closed-loop controller and to reduce extra energy consumption for feedback compensation. In this paper, we focus on developing the motion planning techniques for tracking tasks which realize the above-mentioned concept.

The sensitivity of the robotic arm to disturbances depends on the arm posture, since the dynamics and kinematics of the robotic arm vary according to the arm posture. Based on the sensitivity analysis theory, the sensitivity to unknown disturbances is measured by singular values and singular vectors of a matrix which represents the input-output relation of a system. In the proposed motion planning, using the sensitivity measure, we find the arm posture whose end-

The authors are with the Department of Mechanical Systems Engineering, National Defense Academy of Japan, 1-10-20, Hashirimizu, Yokosuka, JAPAN $\{$ yamawaki, yashima\}@nda.ac.jp

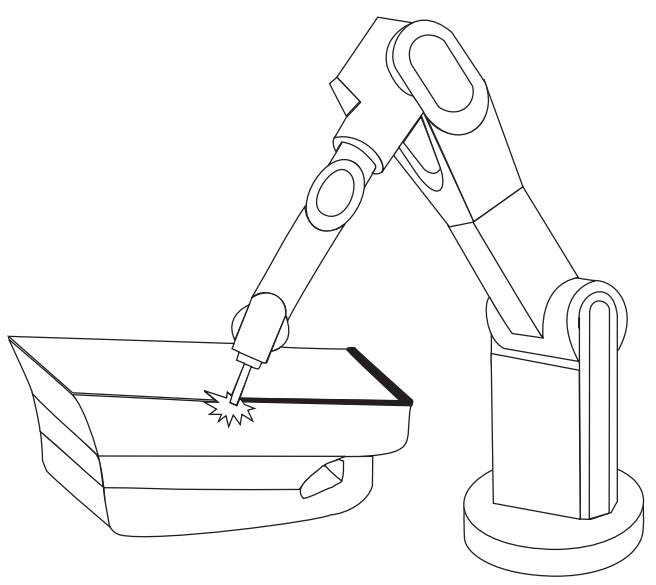

Fig. 1. Tracking tasks such as welding and painting

effector is insusceptible to unknown disturbances in the direction where the tracking task accuracy is required.

There are many planning methods for a robotic arm to find time-optimal motions [1], [8] and collision-free paths [4], [5]. Also, like the proposed method, planning methods based on a matrix analysis, such as the manipulability, the dynamic manipulability, etc., have been proposed [7]. However, these methods have not designed to find arm motions which are insusceptible to unknown disturbances. In this paper, based on our previous work on robust hitting by the robotic arm [10], [11], which finds the arm trajectory insusceptible to unknown disturbances for hitting a stationary object, a novel tracking motion planning method is proposed.

This paper is organized as follows. Section II discusses the sensitivity of the end-effector to unknown disturbances. Section III proposes the novel planning method for the tracking task using the sensitivity measures. In Section IV, the validity of the proposed method is shown by simulations and experiments. Conclusions are found in Section V.

\section{SEnsitivity OF END-EFFECTOR TO DisturbanCES}

\section{A. Model of Robotic Arm}

The equation of motion of a $n$ degree-of-freedom robotic arm can be described as

$$
M(\theta) \ddot{\theta}+\boldsymbol{h}(\boldsymbol{\theta}, \dot{\theta})+\boldsymbol{g}(\boldsymbol{\theta})=\boldsymbol{\tau}+\boldsymbol{d}
$$

where $\boldsymbol{\theta} \in \Re^{n}$ is the joint position, $\boldsymbol{\tau} \in \Re^{n}$ is the joint torque, $\boldsymbol{d} \in \Re^{n}$ is unknown disturbances, $\boldsymbol{M}(\boldsymbol{\theta}) \in \Re^{n \times n}$ is the inertia matrix, $\boldsymbol{h}(\boldsymbol{\theta}, \dot{\boldsymbol{\theta}}) \in \Re^{n}$ is the centrifugal force and the Coriolis force, and $\boldsymbol{g}(\boldsymbol{\theta}) \in \Re^{n}$ is the gravitational force. 
Let the Cartesian coordinate position of the end-effector be $\boldsymbol{p} \in \Re^{m}(m \leq 3)$. A kinematic relation between $\boldsymbol{\theta}$ and $\boldsymbol{p}$ can be described as

$$
p=f(\theta)
$$

Friction, mechanical backlash, etc., which are not shown explicitly in the robotic arm model, are applied to the robotic arm as unknown disturbances. Using a linear system theory, the sensitivity of the end-effector to unknown disturbances can be analyzed.

We regard (1) and (2) with $\boldsymbol{d}=0$ as the nominal model of the robotic arm. Since the robotic arm generally executes tracking tasks such as welding, painting, etc. with low speed, it is assumed that the centrifugal force and the Coriolis force do not so much affect the tracking tasks. We also assume that the physical parameters of the robotic arm model are found by parameter identification.

Linearizing the nominal model with respect to equilibrium points yields the state equation and output equation of the robotic arm with $n$ inputs and $m$ outputs, as follows:

$$
\begin{aligned}
\frac{d}{d t}\left[\begin{array}{c}
\Delta \boldsymbol{\theta}(t) \\
\Delta \dot{\boldsymbol{\theta}}(t)
\end{array}\right] & =\boldsymbol{A}\left[\begin{array}{c}
\Delta \boldsymbol{\theta}(t) \\
\Delta \dot{\boldsymbol{\theta}}(t)
\end{array}\right]+\boldsymbol{B} \Delta \boldsymbol{\tau}(t) \\
\Delta \boldsymbol{p}(t) & =\boldsymbol{C}\left[\begin{array}{c}
\Delta \boldsymbol{\theta}(t) \\
\Delta \dot{\boldsymbol{\theta}}(t)
\end{array}\right]
\end{aligned}
$$

where

$$
\boldsymbol{A}=\left[\begin{array}{cc}
\mathbf{0} & \boldsymbol{I}_{n} \\
-\boldsymbol{M}^{-1} \boldsymbol{G} & \mathbf{0}
\end{array}\right], \quad \boldsymbol{B}=\left[\begin{array}{c}
\mathbf{0} \\
\boldsymbol{M}^{-1}
\end{array}\right], \quad \boldsymbol{C}=\left[\begin{array}{ll}
\boldsymbol{J} & 0
\end{array}\right],
$$

$\boldsymbol{J}(\boldsymbol{\theta})=\partial \boldsymbol{f} / \partial \boldsymbol{\theta} \in \Re^{m \times n}$ is Jacobian matrix and $\boldsymbol{G}(\boldsymbol{\theta})=$ $\partial \boldsymbol{g} / \partial \boldsymbol{\theta} \in \Re^{n \times n}$.

\section{B. Sensitivity Measure}

A system $(\boldsymbol{A}, \boldsymbol{B}, \boldsymbol{C})$ is said to be output controllable if it is possible to construct control inputs that will transfer any given initial output to any final output until a finite time interval. The characteristics are shown by an output controllability matrix

$$
\boldsymbol{N}=\boldsymbol{C}\left[\boldsymbol{B} \boldsymbol{A B} \cdots \boldsymbol{A}^{2 n-1} \boldsymbol{B}\right] .
$$

Substituting (5) into (6) yields the output controllability matrix of the robotic arm, which relates the set of output controllable positions of the end-effector to the set of applied control inputs for a finite time interval.

According to the sensitivity analysis theory [3], the maximum and minimum sensitivity of the end-effector's motion to joint torques $\widehat{\tau}$ with unknown disturbances for a finite time interval are given respectively by

$$
\max _{\widehat{\boldsymbol{\tau}} \neq 0} \frac{\|\boldsymbol{N} \widehat{\boldsymbol{\tau}}\|}{\|\widehat{\boldsymbol{\tau}}\|}=\sigma_{\max }, \quad \min _{\widehat{\boldsymbol{\tau}} \neq 0} \frac{\|\boldsymbol{N} \widehat{\boldsymbol{\tau}}\|}{\|\widehat{\boldsymbol{\tau}}\|}=\sigma_{\min }
$$

where $\sigma_{\max }$ and $\sigma_{\min }$ are the maximum singular value and the minimum singular value of the output controllability matrix, respectively. The singular values $\sigma_{\max }$ and $\sigma_{\min }$ point to the corresponding orthogonal singular vectors.

Since the end-effector is most subject to unknown disturbances in the direction of the maximum singular vector, its

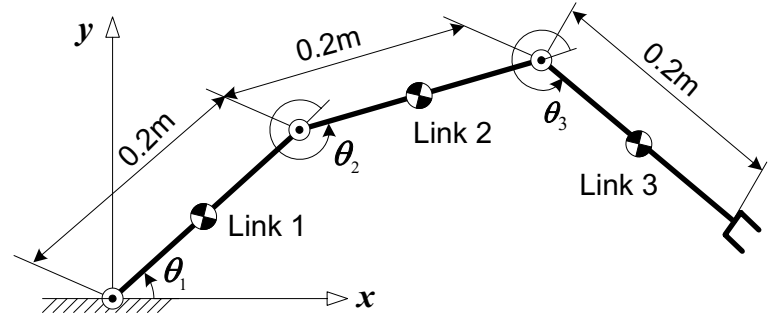

Fig. 2. Three-revolute-joint robotic arm which moves within a horizontal plane. The maximum values of the diagonal element of the inertia matrix $\boldsymbol{M}$ are $(1.880,0.200,0.024) \mathrm{kgm}^{2}$.

positions are distributed along near the maximum singular vector due to the disturbances. In contrast, since the endeffector is least subject to unknown disturbances in the direction of the minimum singular vector, the distributions of its positions are most suppressed along the direction.

Meanwhile, the degrees of distribution of the endeffector's position errors along each singular vector are shown by the condition number of the output controllability matrix as

$$
c=\sigma_{\max } / \sigma_{\min }
$$

The larger the condition number is, the more the endeffector's position errors converge in the direction of the maximum singular vector than in the other directions. In contrast, the smaller the condition number is, the more the end-effector's position errors are distributed in an entire space.

In this manner, the sensitivity of the end-effector to unknown disturbances is evaluated by the direction of the singular vectors and the condition number. Next, we show the above-mentioned validity with some simulation examples and discuss the application to a motion planning.

\section{Simulation Examples}

Fig. 2 shows the model of a three-revolute-joint robotic arm that moves within a horizontal plane, which corresponds to an actual robotic arm shown in Fig. 11.

Inputting disturbance torques to the robotic arm at rest for an infinitesimal time $t_{f}$, we calculate the distribution of the end-effector's position error caused by the disturbance using (1) and (2). This simulation is repeated 50 times. We assume that the disturbance torques are white noise whose average is zero and variance is $4 \mathrm{~N}^{2} \mathrm{~m}^{2}$, and $t_{f}=0.2 \mathrm{sec}$.

As shown in Fig. 3 (a), let the initial end-effector's position and joint angle be $\boldsymbol{p}=(0.15,0.45) \mathrm{m}$ and $\boldsymbol{\theta}=$ $(127.3,-73.9,-12.6)^{T}$ deg, respectively. Fig. 3 (b) shows the distribution of the end-effector's position error caused by the disturbance torques. The center of this figure indicates the initial end-effector's position. The straight lines indicate the directions of the maximum and minimum singular vectors at the initial arm posture. The distributions of the end-effector's position are denoted by $\times$.

As seen from the simulation result, the distribution of the end-effector's positions mostly converge along near the maximum singular vector, while the distribution in the direction 


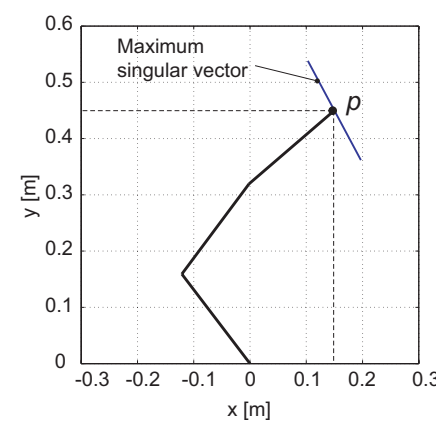

(a)

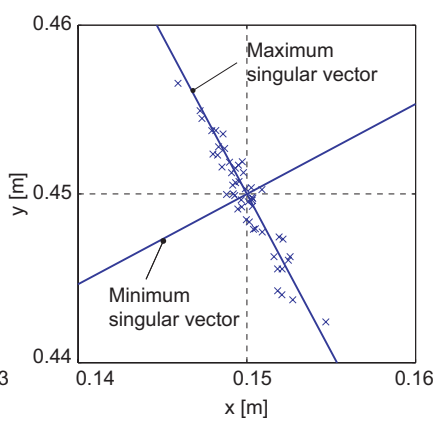

(b)
Fig. 3. (a) Arm posture. (b) Position error distribution.

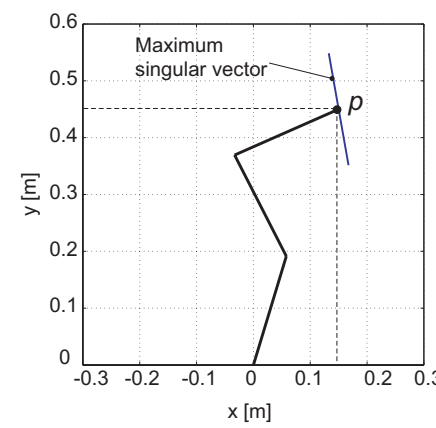

(a)

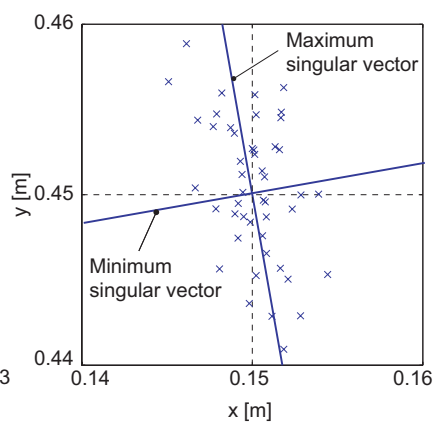

(b)
Fig. 4. (a) Arm posture. (b) Position error distribution.

of the minimum singular vector, which is orthogonal to the maximum singular vector, are suppressed.

Next, as shown in Fig. 4 (a), we consider a different arm posture with the same initial end-effector's position shown in Fig. 3 (a), where the joint angle is $\boldsymbol{\theta}=$ $(73.1,43.9,-93.3)^{T} \mathrm{deg}$. The directions of the singular vectors change significantly and the condition number reduces from 5.76 to 3.30. As seen from Fig. 4 (b), the end-effector's position errors are distributed more wider than that of Fig. 3 (b) since the condition number becomes smaller.

\section{Application to Motion Planning}

Since the output controllability matrix of (6) involves the matrices $\boldsymbol{J}(\boldsymbol{\theta})$ and $\boldsymbol{M}(\boldsymbol{\theta})$, the directions of the singular vectors depend on the arm posture.

All tasks do not require fully degrees of freedom in the Cartesian space. The robotic arm can have an infinite arm posture using the redundancy for a specified task. Therefore, the direction of the singular vector can be set arbitrarily by changing the arm posture under the condition that the arm's dynamics and kinematics are satisfied, which is shown by a simulation using a three-revolute-joint robotic arm in Fig. 5, where the straight lines at the end-effector indicate the directions of the maximum singular vectors. It is found that the arm's redundancy makes it possible to change the direction in which the end-effector is subject to the unknown disturbances by changing the arm posture.

Taking into consideration the relationship between the

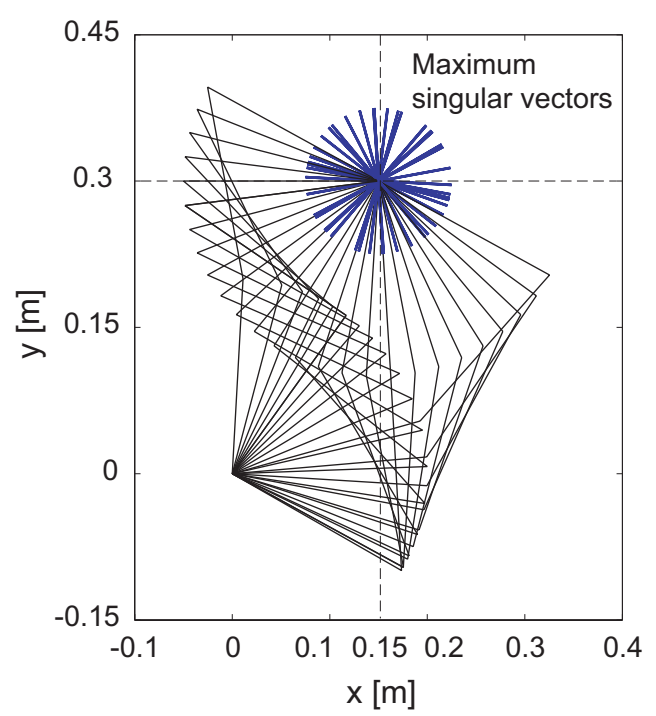

Fig. 5. Change of singular vectors according to the change of arm posture.

singular vectors and end-effector's position error, we propose a novel motion planning method for a tracking task by the robotic arm, which finds the arm posture that is insusceptible to unknown disturbances. Changing arm postures to obtain arm's dynamics and kinematics which are insusceptible to unknown disturbances is called dynamics shaping [10].

We consider that errors perpendicular to the direction of a desired end-effector's motion are more important in tracking tasks such as welding and painting since such errors cause collision with a workpiece. The basic idea of the proposed motion planning is to find the arm posture from the beginning to the end of the tracking task not only so that the maximum singular vector points toward the direction of a desired endeffector's path but so that the condition number becomes larger to make the end-effector's error converge along the maximum singular vector as much as possible. Using the obtained arm trajectory as a reference trajectory in the trajectory tracking system, we can obtain the arm motion so that the end-effector will not deviate greatly from the desired end-effector's path even though unknown disturbances are applied to the robotic arm.

In the next section, we detail the motion planning method for a tracking task by a three-revolute-joint robotic arm.

\section{Motion Planning FOR TRACKING TASK}

This section proposes a motion planning method for the tracking task, which finds the arm motion so that the maximum singular vector points toward the direction of the specified end-effector's path as much as possible. The proposed motion planning consists of three phases as shown below.

\section{A. Phase 1: Planning of Initial Arm Posture}

We find an initial arm posture whose maximum singular vector points to the direction of the desired end-effector's path. A randomized algorithm, which has high performance 


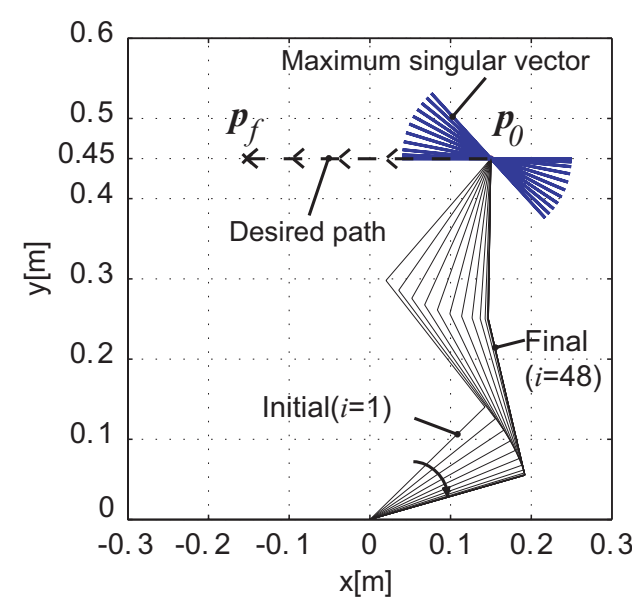

(a) Phase 1

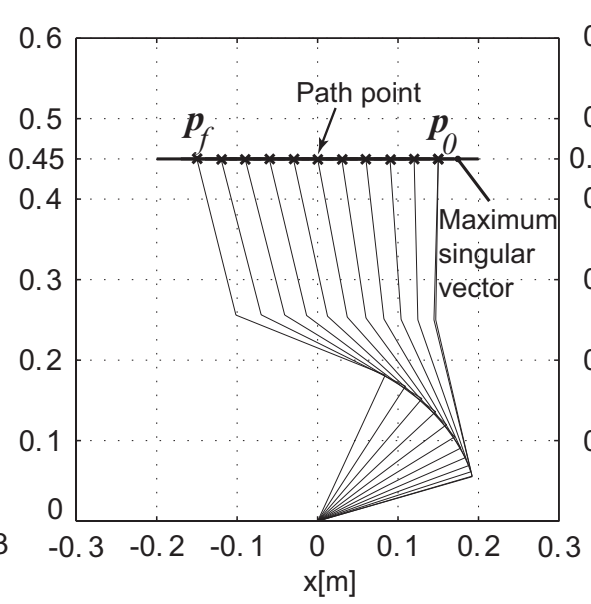

(b) Phase 2

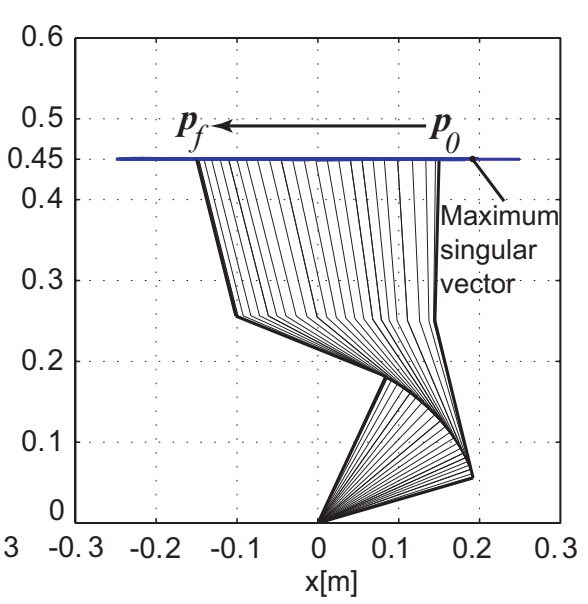

(c) Phase 3

Fig. 6. (a) Phase 1 obtains the initial arm posture. (b) Phase 2 obtains arm posture at each path point of end-effector. (c) Phase 3 finds arm trajectory which takes arm posture at each path point obtained in phase2.

for a global searching, is applied. The algorithm is given as follows, where $i$ denotes the iteration number of a search.

1) As a global search, we select the first joint angle $\theta_{1}$ at random in a specified range.

2) As a local search, we set an infinitesimal angle $\Delta \theta_{1} \in$ $\left\{-\varepsilon_{\theta}, 0, \varepsilon_{\theta}\right\}$ and get new three joint angles $\theta_{1}$ around the previously obtained joint angle $\theta_{1}$ in step 1 by using the following equation

$$
\theta_{1} \leftarrow \theta_{1}+\Delta \theta_{1}
$$

We find $\theta_{2}$ and $\theta_{3}$ so that end-effectors can be located at the desired initial position for the newly set $\theta_{1}$. A total of three arm postures are obtained.

3) For each arm posture obtained in step 2, we calculate a deviation angle $q=\cos ^{-1}\left(\boldsymbol{u}_{\max }^{T} \boldsymbol{u}_{t}\right)$ between its maximum singular vector, $\boldsymbol{u}_{\max }$, and the direction of the desired path, $\boldsymbol{u}_{t}$. If the minimum $q$ among them satisfies $|q|<\left|q^{(i-1)}\right|$, where $q^{(i-1)}$ is the deviation angle of the previous search step, we update the arm posture as an initial arm posture candidate. Otherwise, we go back to step 1 .

4) If the deviation angle satisfies $\left|q^{(i)}\right|<\epsilon$, we stop searching and set the arm posture as the initial arm posture.

5) Updating the iteration number $i$, if $i>i_{\max }$ then we terminate searching. Otherwise, we go back to step 2 .

If we can get multiple candidates for the initial arm posture in this phase, we choose an arm posture which has the largest condition number.

Fig. 6 (a) shows simulation results which reveal the transition of the arm posture by the iteration. We set the initial and final points of the end-effector to $\boldsymbol{p}_{0}=(0.15,0.45)^{T} \mathrm{~m}$, $\boldsymbol{p}_{f}=(-0.15,0.45)^{T} \mathrm{~m}$, respectively, and set parameters to $\varepsilon_{\theta}=q / 25 \mathrm{deg}$ and $\epsilon=0.001 \mathrm{deg}$. The desired end-effector's path is a straight line connecting $\boldsymbol{p}_{0}$ and $\boldsymbol{p}_{f}$, which is parallel to the $x$-axis. We can see that the maximum singular vectors approach to the direction of the desired path by repeating the search. The initial arm posture was found at the 48th trial.

\section{B. Phase 2: Planning of Arm Posture at Each Path Point of End-effector}

We set path points on the desired end-effector's path and find arm postures at each path point from the initial path point to the final point of the end-effector by using a similar approach to the phase 1. We show an algorithm to find an arm posture at the $k$-th path point below.

1) As a global search, we set the first joint angle $\theta_{1, k}$ at the $k$ th path point by using the following equation

$$
\theta_{1, k} \leftarrow \theta_{1, k-1}+\Delta \widehat{\theta}_{1}
$$

where $\theta_{1, k-1}$ is a known first joint angle at the $k$-1th path point and $\Delta \widehat{\theta}_{1}$ is chosen at random in a range $\left\{-\Delta \widehat{\theta}_{\max } \leq \Delta \widehat{\theta}_{1} \leq \Delta \widehat{\theta}_{\max }\right\}$. The upper and lower bounds of the range, $\pm \Delta \widehat{\theta}_{\max }$, are given by taking into consideration the joint motor's performance.

2) As a local search, we set an infinitesimal angle $\Delta \theta_{1} \in$ $\left\{-\varepsilon_{\theta}, 0, \varepsilon_{\theta}\right\}$ and obtain new three joint angles $\theta_{1, k}$ around the previously obtained joint angle $\theta_{1, k}$ in step 1 by using the following equation

$$
\theta_{1, k} \leftarrow \theta_{1, k}+\Delta \theta_{1}
$$

We find $\theta_{2, k}$ and $\theta_{3, k}$ so that end-effectors can be located at the $k$ th path point for the newly set $\theta_{1, k}$, taking into consideration the continuity of each joint angle between each path point. A total of three arm postures at most are obtained.

3) For each arm posture obtained in step 2, we calculate a deviation angle $q=\cos ^{-1}\left(\boldsymbol{u}_{\max }^{T} \boldsymbol{u}_{t}\right)$ between its maximum singular vector, $\boldsymbol{u}_{\max }$, and the direction of the desired path, $\boldsymbol{u}_{t}$. If the minimum $q$ among them satisfies $|q|<\left|q^{(i-1)}\right|$, then we update the arm posture as an arm posture candidate at the $k$ th path point. Otherwise, we go back to step 1 .

4) If the deviation angle satisfies $\left|q^{(i)}\right|<\epsilon$, we stop searching and set the arm posture candidate as the arm posture at the $k$ th path point. 


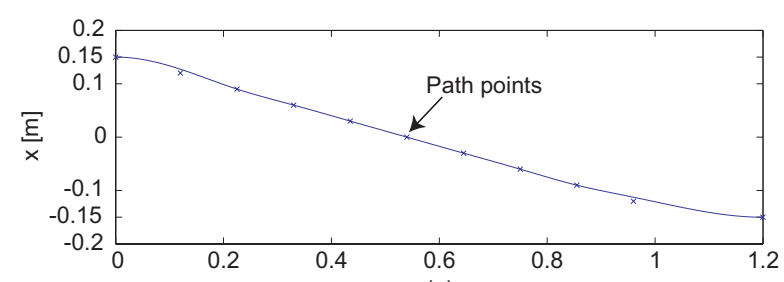

(a)

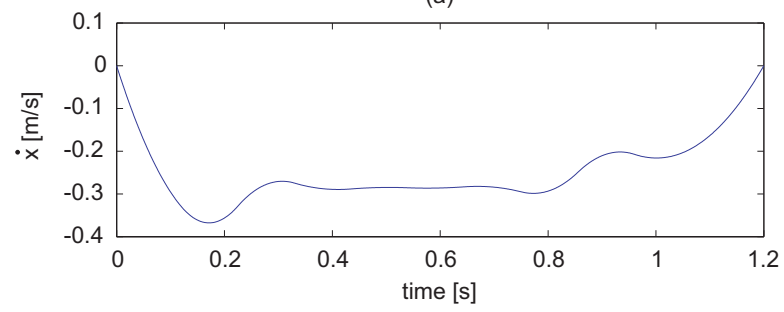

(b)

Fig. 7. Position and velocity trajectories of end-effector in the $x$ direction

5) Updating the iteration number $i$, if $i>i_{\max }$ then we terminate searching. Otherwise, we go back to step 2.

If we can get multiple candidates for the arm posture in this phase, we choose an arm posture which has the largest condition number.

Fig. 6 (b) shows arm postures and the corresponding maximum singular vectors at ten path points set on the desired end-effector's path. We can see that the arm postures are found so that the maximum singular vectors can point to the direction of the desired end-effector's path.

\section{Phase 3: Arm Trajectory Planning}

Phase 3 generates the trajectories of the joint angles and torques which move the robotic arm from the initial to the final in a given time $t_{f}$, taking the arm posture at each path point obtained in phase 2 as much as possible. To find the arm trajectory, we use a trajectory generation method [6] which generates a trajectory represented by a cubic spline function between path points.

Fig. 6 (c) shows the simulation results. We set the initial and final joint velocity values to zero and $t_{f}=1.2 \mathrm{sec}$. We found an arm trajectory which takes almost the same postures as the specified arm postures at each path point. Fig. 7 shows the position and velocity trajectories of the end-effector in the $x$ direction. The smooth trajectories are obtained.

\section{Simulations And EXPERIMENTS}

\section{A. Simulations}

We show by simulations that the arm trajectory obtained in the previous section makes the end-effector track the desired path as closely as possible even though disturbances are applied to the joint torque.

The input torque is produced by adding disturbance torques to a nominal joint torque trajectory obtained in the phase 3 . We get the locus of the end-effector by repeating the trial 50 times. The disturbance torques are white noise whose average is zero and variance is $4 \mathrm{~N}^{2} \mathrm{~m}^{2}$.

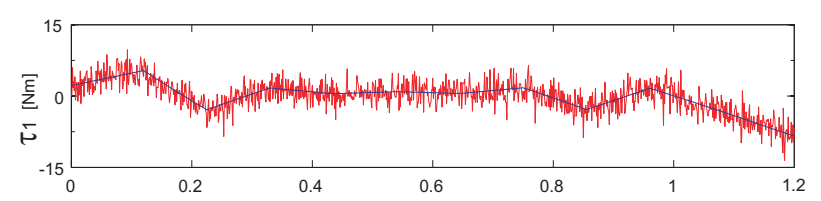

(a)

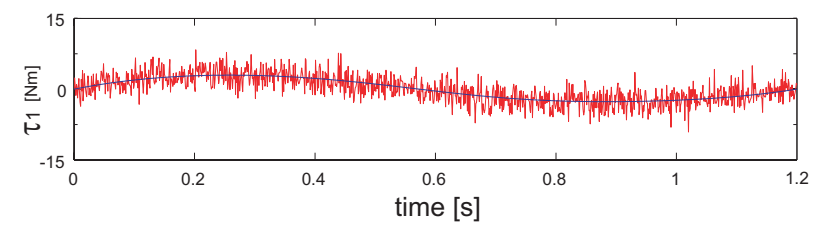

(b)

Fig. 8. Joint torque trajectory. (a) the trajectory obtained by the proposed method. (b) the comparison trajectory.

For comparison, we also conduct similar simulations using another trajectory, whose end-effector's path is the same but the joint angle trajectory is different, which is expressed by a fifth order polynomial about a time.

Figs. 8 (a) and (b) show the input torque trajectories of the first joint with disturbance torques for each case, respectively.

Figs. 9 and 10 describe the simulation results of the loci of the end-effector for each case. The loci of Fig. 9, which is the case of the trajectory obtained by the proposed method, converge closer to the desired end-effector's path parallel to the $x$-axis than that of Fig. 10 . We can see that the obtained trajectory is insusceptible to unknown disturbances.

\section{B. Experiments}

This section verifies experimentally the validity of simulation results. Fig. 11 shows a three-DOF direct-drive arm used in this experiment. The maximum torque of each DD motor are 70, 15 and $15 \mathrm{Nm}$, respectively. The end-effector's path, the physical parameters of the robotic arm, etc. are identical to those used in the simulations. The input torque with disturbance torques, which is obtained in the previous section, is transmitted to the motor drivers without any feedback compensation.

Similar to the simulations shown in Figs. 9 and 10, we conduct two experiments on tracking tasks using the trajectory obtained by proposed method and the comparison trajectory. We get the loci of the end-effector by repeating the tracking tasks 50 times. The loci are obtained from the joint angles measured by encoders.

Figs. 12 (a) and (b) describe the experimental results of the end-effector's loci for the two cases, respectively. Compared Figs. 12 (a) and (b) with Figs. 9 and 10, the tendencies to distribution of the end-effector's position are almost same in the experiments and simulations. We can reaffirm experimentally that the proposed method can find the greatly improved tracking trajectories which are insusceptible to unknown disturbances. It is expected that the proposed techniques can be used as a trajectory generator in a closed-loop tracking system. 


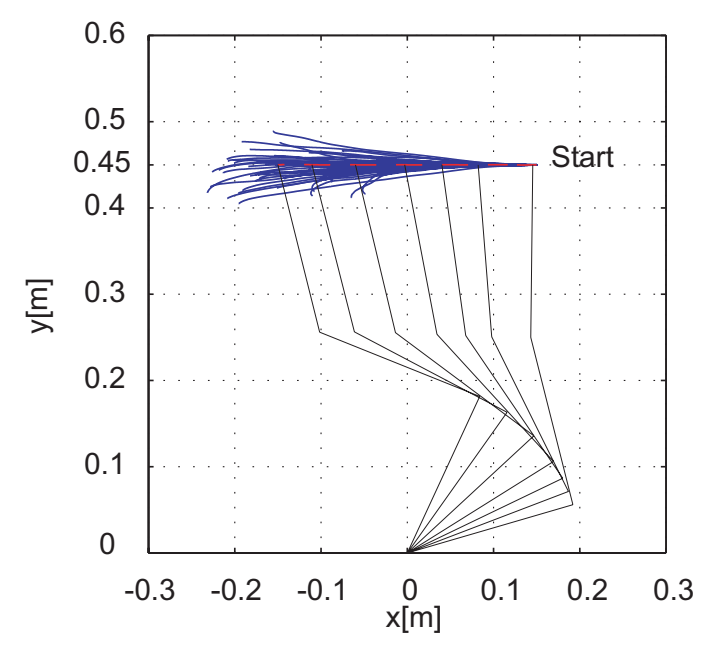

Fig. 9. Simulation results of end-effector distributions for the trajectory obtained by proposed method.

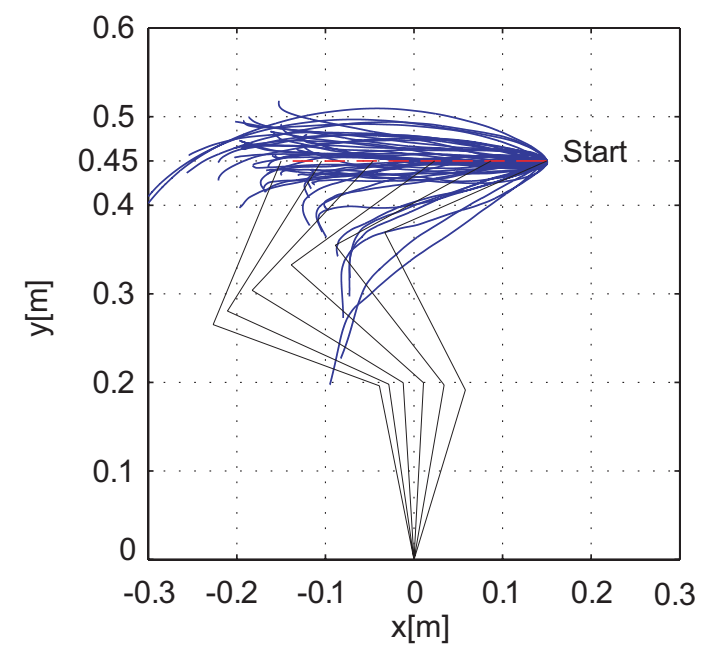

Fig. 10. Simulation results of end-effector distributions for the comparison trajectory.

\section{CONCLUSION}

In the present paper, we showed a novel motion planning method for a robotic arm, which finds the arm trajectory that is insusceptible to unknown disturbances in the direction where task accuracy is required. First, we showed that the sensitivity of the end-effector to unknown disturbances is measured by the direction of singular vectors and the condition number of the robotic arm system. We proposed a motion planning method for tracking tasks, which finds the arm posture not only so that the maximum singular vector points toward the direction of a desired end-effector's path but so that the condition number becomes larger. We showed by simulations and experiments that the end-effector can track the desired path as closely as possible even though disturbances are applied to the robotic arm. Greatly improved tracking trajectory was obtained.

Our future work is to incorporate the proposed techniques
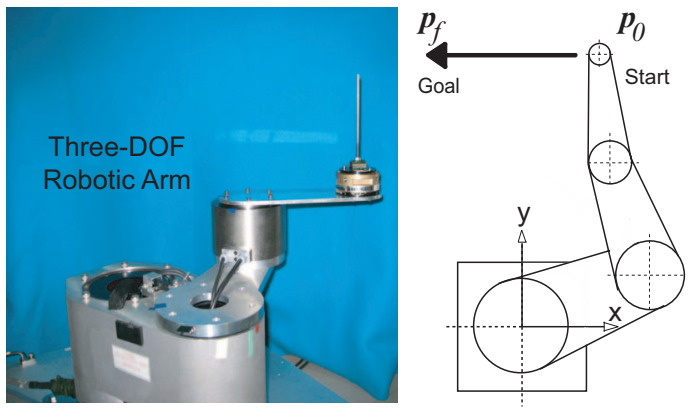

Fig. 11. Overview of three-DOF robotic arm

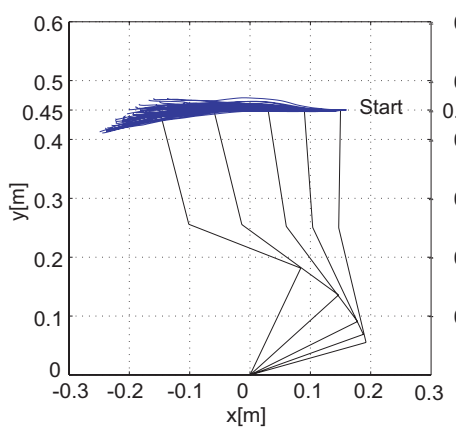

(a)

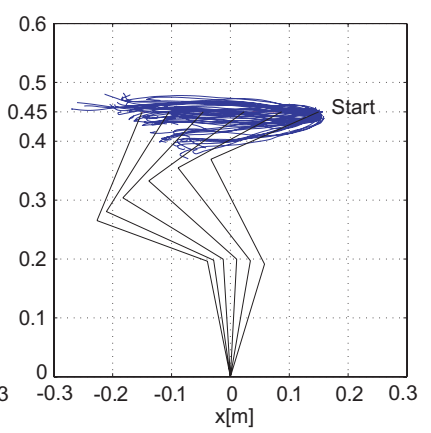

(b)
Fig. 12. Experimental results of end-effector distributions for (a) the trajectory obtained by proposed method and (b) the comparison trajectory.

as a reference trajectory generator into a conventional closedloop tracking controller system in order to design more robust tracking systems in a simple way.

\section{REFERENCES}

[1] J.E. Bobrow, S. Dubowsky, and J.S. Gibson, "Time-optimal Control of Robotic Manipulators Along Specified Paths," Int. J. Robotics Research, vol.4, no. 3, pp. 3-17, 1985.

[2] B-S. Chen, T-S. Lee and J-H. Feng, "A Nonlinear $H_{\infty}$ Control Design in Robotic Systems under Parameter Perturbation and External Disturbance," Int. J. of Control, vol.59, no.2, pp.439-461, 1994.

[3] G.H. Golub and C.F. Van Loan, Matrix Computations, The Johns Hopkins University Press, 1996.

[4] L.E. Kavraki, P. Svestka, J-C. Latombe, and M.H. Overmars, "Probabilistic Roadmaps for Path Planning in High-Dimensional Configuration Spaces," IEEE Trans. on Robotics and Automation, vol. 12, no. 4, pp. 566-580, 1996

[5] P. Leven and S. Hutchinson, "Using Manipulability to Bias Sampling During the Construction of Probabilistic Roadmaps," IEEE Trans. on Robotics and Automation, vol. 19, no. 6, pp. 1020-1026, 2003.

[6] C-S. Lin, P-R. Chang and J.Y.S. Luh, "Formulation and Optimization of Cubic Polynomial Joint Trajectories for Industrial Robots," IEEE Trans. on Automatic Control, vol.AC28, no.12, pp.1066-1074, 1983.

[7] Y. Nakamura, Advanced Robotics, Addison Wesley, 1991.

[8] Z. Shiller and S. Dubowsky, "On Computing the Global Time-optimal Motions of Robotic Manipulators in the Presence of Obstacle," IEEE Trans. on Robotics and Automation, vol. 7, no. 6, pp. 785-797, 1991.

[9] M.W. Spong, "On the Robust Control of Robot Manipulators," IEEE Trans. on Automatic Control, vol.AC37, pp.1782-1786, 1992.

[10] T. Yamawaki and M. Yashima, "Robust Hitting with Dynamics Shaping," Proc. of the 2009 IEEE Int. Conf. on Robotics and Automation, pp. 114-119, 2009.

[11] T. Yamawaki and M. Yashima, "Arm Trajectory Planning by Controlling the Direction of End-point Position Error Caused by Disturbance," Proc. of the 2008 IEEE/ASME Int. Conf. on Advanced Intelligent Mechatronics, pp. 1120-1125, 2008. 